\title{
Improvement of Ion Confinement in Core Electron-Root Confinement (CERC) Plasmas in Large Helical Device
}

\author{
Yasuhiko TAKEIRI, Masayuki YOKOYAMA, Kenichi NAGAOKA, Katsumi IDA, Shin KUBO, \\ Takashi SHIMOZUMA, Hisamichi FUNABA, Masaki OSAKABE, Katsunori IKEDA, \\ Katsuyoshi TSUMORI, Yashihide OKA, Mikiro YOSHINUMA, Shigeru MORITA, \\ Motoshi GOTO, Kazumichi NARIHARA, Ichihiro YAMADA, Kenji TANAKA, \\ Osamu KANEKO, Akio KOMORI and LHD Experimental Group \\ National Institute for Fusion Science, 322-6 Oroshi-cho, Toki 509-5292, Japan
}

(Received 25 November 2007 / Accepted 7 March 2008)

\begin{abstract}
An increase in ion temperature has been observed with superposition of centrally focused electron cyclotron resonance heating $(\mathrm{ECRH})$ to plasmas heated by high-energy neutral beam injection (NBI) in Large Helical Device. The ion-temperature $\left(T_{\mathrm{i}}\right)$ rise is accompanied by the formation of electron internal transport barrier (ITB). A transport analysis shows that ion transport as well as electron transport is improved with the reduction of anomalous transport. A neoclassical ambipolar flux calculation shows a positive radial-electric field $\left(E_{\mathrm{r}}\right)$ in the region of the $T_{\mathrm{i}}$ rise, and $E_{\mathrm{r}}$ should suppress the enhancement of ripple transport due to the $T_{\mathrm{i}}$-rise. These analyses indicate the ion transport improvement in the core electron-root confinement plasmas. Toroidal rotation is driven in the co-direction by applying ECRH, and the toroidal rotation velocity is increased with the $T_{\mathrm{i}}$ rise. A correlation between the $T_{\mathrm{i}}$ rise and toroidal rotation is suggested.
\end{abstract}

(c) 2008 The Japan Society of Plasma Science and Nuclear Fusion Research

Keywords: core electron-root confinement, neoclassical transport, anomalous transport, radial electric field, ion transport, toroidal rotation, electron internal transport barrier

DOI: $10.1585 /$ pfr.3.S1031

\section{Introduction}

Helical devices have non-axisymmetric magnetic field configuration, which induces neoclassical ripple transport. Neoclassical ion and electron fluxes are strongly dependent on the radial electric field $\left(E_{\mathrm{r}}\right)$, whose value is determined by the ambipolarity condition of these fluxes. $E_{\mathrm{r}}$ also has a great influence on the plasma properties related to both ripple and anomalous transport. In major middlesized helical devices such as CHS, W7-AS, and TJ-II, the improvement of electron transport is commonly observed in core electron-root confinement (CERC) plasmas [1,2], in which a large positive $E_{\mathrm{r}}$ is observed in the core region. The CERC plasmas are obtained when the central electrons are strongly heated with electron cyclotron resonance heating (ECRH), and they are also observed in Large Helical Device (LHD) [3-5]. In the CERC plasmas, an electron internal transport barrier (electron-ITB) with a steep gradient of the electron-temperature $\left(T_{\mathrm{e}}\right)$ profile is formed in the core region, and the core electron transport is improved in the neoclassical electron root with the positive $E_{\mathrm{r}}[1,2,6]$. As a result, high $T_{\mathrm{e}}$ is achieved in the CERC plasmas.

The confinement improvement in the neoclassical electron root is specific to helical systems, and ion transport should also be improved in the CERC plasmas according to a theoretical prediction. We have observed an ion-

author'se-mail: takeiri@nifs.ac.jp temperature $\left(T_{\mathrm{i}}\right)$ rise in the neutral beam injection (NBI) plasma by applying centrally focused ECRH in LHD, in which the $T_{\mathrm{e}}$ profile shows electron-ITB formation. It is thought that the $T_{\mathrm{i}}$ rise is ascribed to the ion transport improvement in the CERC plasmas. A transport analysis and a comparison with the neoclassical calculation are carried out, and the results are discussed. The properties of the CERC plasmas, which are realized by applying ECRH to the NBI plasmas, are presented with a view of ion transport improvement.

\section{NBI and ECRH Systems}

LHD is the world's largest superconducting helical device [7], and it is equipped with NBI and ECRH systems for plasma heating. The NBI system consists of three high-energy negative-ion-based NB injectors $[8,9]$ and a low-energy positive-ion-based NB injector [10]. The arrangement of the NBI system is illustrated in Fig. 1. The injection direction of the negative-NBI is tangential while that of the positive-NBI is perpendicular to the magnetic axis. The injection energy of the negative-NBI is as high as $180 \mathrm{keV}$ and plasma electrons are dominantly heated. The total injection power achieved is $14 \mathrm{MW}$ in the negativeNBI. To increase the ion heating power effectively with the negative-NBI, high- $Z$ discharges are utilized in high$T_{\mathrm{i}}$ experiments with $\mathrm{Ar} / \mathrm{Ne}$ gas puffing [11]. As a result, $T_{\mathrm{i}}$ is increased with an increase in ion heating power and 


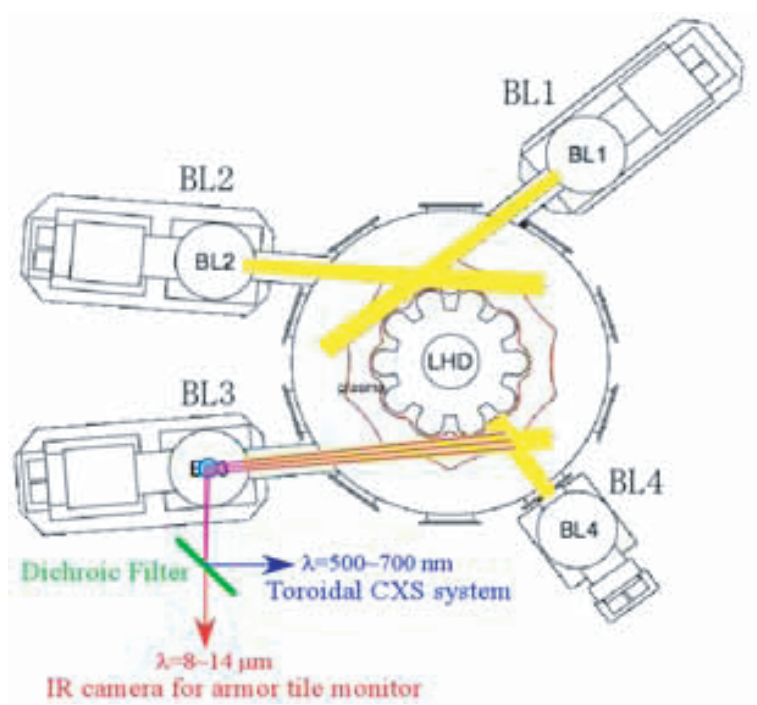

Fig. 1 Arrangement of three high-energy tangential NB injectors (BL1, BL2, and BL3) and a low-energy perpendicular NB injector (BL4). A toroidal line of sight from the BL3-injection port for the CXS system, which utilizes the BL4 beam, is also indicated.

reached $13.5 \mathrm{keV}$ [12]. For efficient ion heating in hydrogen plasmas, the positive-NBI has been recently constructed with an injection energy of $40 \mathrm{keV}$, which dominantly heats plasma ions, and $6 \mathrm{MW}$ of injection power was achieved [10]. The positive-NBI is also utilized for a $T_{\mathrm{i}}$-profile measurement by charge-exchange spectroscopy (CXS) along a toroidal line of sight [13,14], which is better for the measurement in the central region than that along a poloidal line of sight. With this arrangement, toroidal rotation is also measured.

The ECRH system employs 168,84 , and $82.7 \mathrm{GHz}$ gyrotrons [15]. Each microwave is injected as a highly focused Gaussian beam using vertical and horizontal antenna systems with quasi-optical mirrors. The beam-waist radius at the focal point is $15-30 \mathrm{~mm}$. The focus location is variable at $3.5-3.9 \mathrm{~m}$ of the major radius on the equatorial plane. The total injection power achieved is $2.1 \mathrm{MW}$. In the experiments, the second-harmonic heating with 84 and $82.7 \mathrm{GHz}$ microwaves is used at around $1.5 \mathrm{~T}$ of the magnetic field strength on the axis.

\section{Ion Temperature Rise in the CERC Plasmas}

CERC is a specific feature commonly observed in helical systems, and no counterpart is observed in tokamaks. In LHD, by applying centrally focused ECRH to the NBI plasma, a strongly peaked $T_{\mathrm{e}}$ profile is observed in the core region. Such a kind of the electron-ITB formation indicates improvement of electron transport, and reduction of electron thermal diffusivities is recognized together with positive radial electric field $\left(E_{\mathrm{r}}\right)$. This means that the improvement of electron transport in the core region oc-
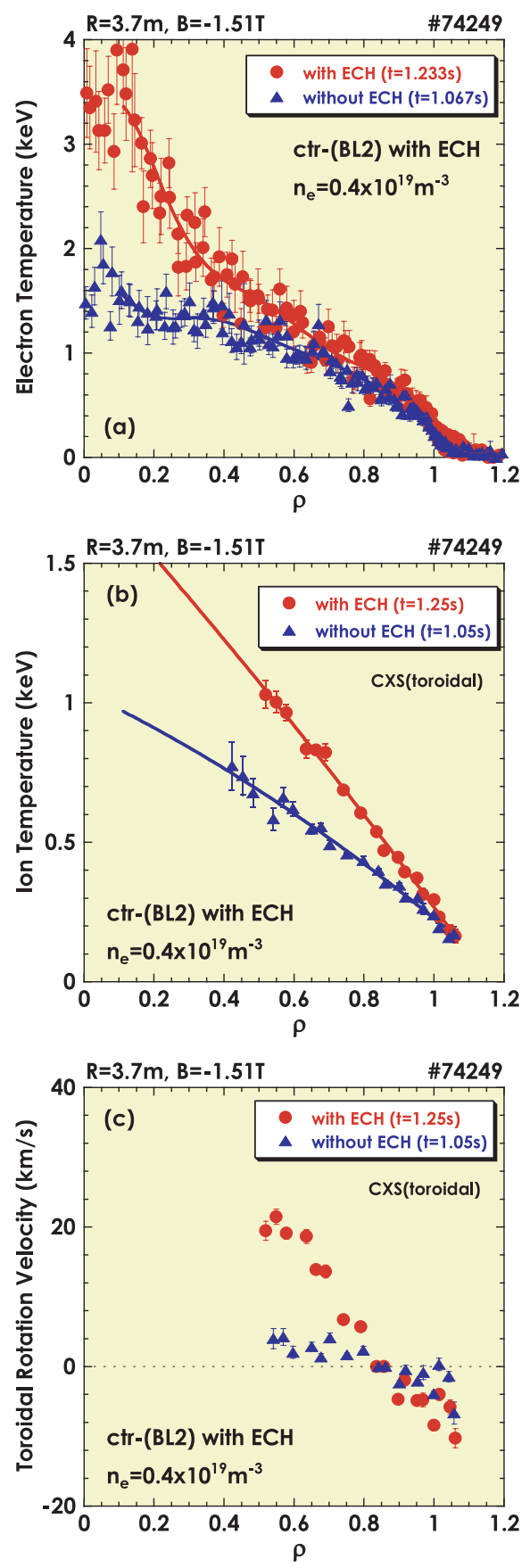

Fig. 2 (a) Electron temperature profiles, (b) ion temperature profiles, and (c) toroidal rotation velocity profiles, with and without ECRH superposition to the plasmas heated with both the counter-NBI (BL2) and the perpendicular-NBI (BL4). While the electron temperature profiles are polynomially fitted, the ion temperature profiles are parabolically extrapolated to the center.

curs in the neoclassical electron root. Ion transport is also expected to be improved in the electron root, and ECRH was applied to plasmas heated by both the negative-NBI and the positive-NBI.

Figure 2 (a) and (b) shows the electron and ion temperature profiles, respectively, in the plasmas with and without the ECRH superposition. The electron density is 
$0.4 \times 10^{19} \mathrm{~m}^{-3}$, and the target plasma is sustained by the counter-NBI (BL2) and the perpendicular-NBI (BL4). The applied ECRH power is $0.68 \mathrm{MW}$ and is focused at about $\rho=0.15$. By applying ECRH, the $T_{\mathrm{e}}$ profile indicates a steep gradient inside $\rho=0.4$, which shows improvement of electron confinement in the electron root. $T_{\mathrm{i}}$ is also increased along with the $T_{\mathrm{e}}$ rise, as shown in Fig. 2 (b). The ion temperature is measured using the charge exchange emission of CVI, and the carbon impurity profile in the core region is strongly hollowed due to the impurity pumpout effect when ECRH is applied. As a result, the central $T_{\mathrm{i}}$ profile is not measured. Although it is unknown how much the central $T_{\mathrm{i}}$ is increased by the ECRH superposition, a $T_{\mathrm{i}}$ rise is observed in the mid-radius region. It seems that the $T_{\mathrm{i}}$-rise location is different from the $T_{\mathrm{e}}$-rise location.

Toroidal rotation is also measured by CXS in a tangential line of sight. Figure 2 (c) shows the toroidal rotation velocity, $V_{\mathrm{t}}$, for the plasmas with and without applying ECRH. It is found that the toroidal rotation is driven in the co-direction at the $T_{\mathrm{i}}$-rise location by applying ECRH. The increase in the toroidal rotation seems to be correlated with the $T_{\mathrm{i}}$ rise, and the spontaneous toroidal rotation due to applying ECRH is suggested to cause the transport improvement.

A transport analysis based on power balance was performed for the plasmas with and without applying ECRH shown in Fig. 2. Since $T_{\mathrm{i}}$ data in the central region is not available, the $T_{\mathrm{i}}$ profiles are parabolically extrapolated to the center using the measured data, as shown in Fig. 2 (b). Thus, ion transport in the central region is not discussed here. As for electron transport, since the heat exchange between electrons and ions is almost negligible in such low-density plasmas, the electron thermal diffusivity is obtained in the entire region. $Z_{\text {eff }}$ is usually 2 to 3 in hydrogen plasmas, and the $Z_{\text {eff }}$ profile should be changed due to the impurity pump-out effect in the central region with the ECRH superposition. The $Z_{\text {eff }}$ profile change would influence the injected neutral-beam deposition (ionization) profile. In LHD, the beam deposition power is estimated using a shine-through power measurement on the beamfacing armor plate [16], and no distinct change of the beam deposition power was observed with the ECRH superposition. This is probably because $Z_{\text {eff }}$ in the NBI plasmas without the ECRH superposition is not high, and the influence of the $Z_{\text {eff }}$ change due to the ECRH superposition on the beam deposition is considered to be small. Therefore, the effect of the $Z_{\text {eff }}$ change is not taken into consideration to estimate the beam deposition profile.

Figures 3 (a) and (b) show the thermal diffusivities for electrons and ions normalized by the gyro-Bohm factor of $T_{\mathrm{e}}^{3 / 2}$ and $T_{\mathrm{i}}^{3 / 2}$, respectively. $\chi_{\mathrm{e}} / T_{\mathrm{e}}^{3 / 2}$ and $\chi_{\mathrm{i}} / T_{\mathrm{i}}^{3 / 2}$ are regarded as measures of the degree of anomalous transport for electrons and ions, respectively. As shown in Fig. 3, by applying ECRH, the thermal diffusivity normalized by the gyro-Bohm factor for ions is reduced in the region of $\rho>0.5$ while that for electrons is not changed in the same
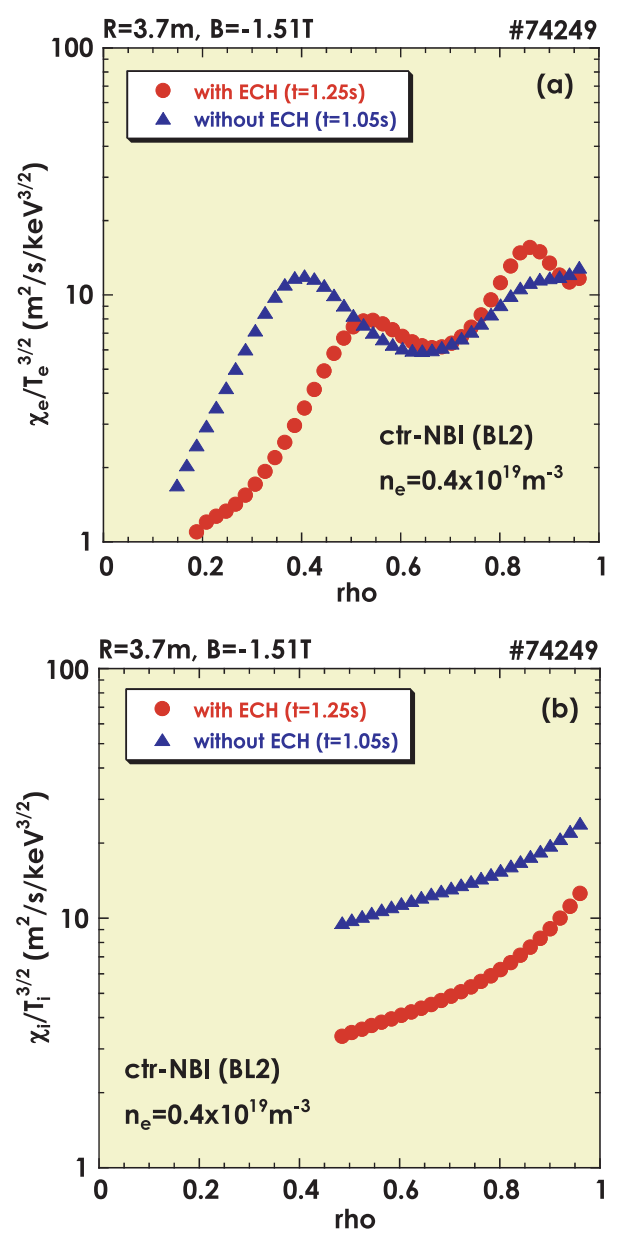

Fig. 3 Profiles of (a) electron thermal diffusivities normalized by $T_{\mathrm{e}}^{3 / 2}, \chi_{\mathrm{e}} / T_{\mathrm{e}}^{3 / 2}$, and (b) ion thermal diffusivities normalized by $T_{\mathrm{i}}^{3 / 2}, \chi_{\mathrm{i}} / T_{\mathrm{i}}^{3 / 2}$, for the NBI plasmas with and without the ECRH superposition shown in Fig. 2.

region. In the central region of $\rho<0.5, \chi_{\mathrm{e}} / T_{\mathrm{e}}^{3 / 2}$ is reduced by applying ECRH, although the change in ion transport in the central region is unknown. These results suggest that the location of the transport improvement by applying ECRH is different between electrons and ions.

The neoclassical calculation was also performed for the plasmas shown in Fig. 2. The GSRAKE code was used [17], and the calculated ambipolar $E_{\mathrm{r}}$ is shown in Fig. 4 (a). Positive $E_{\mathrm{r}}$ is found at around $\rho=0.6$, which corresponds to the $T_{\mathrm{i}}$-rise location. The value of the positive $E_{\mathrm{r}}$ is not large, and the obtained electron root is a single solution. The calculated ion thermal diffusivities, $\chi_{\mathrm{i}}$, including the $E_{\mathrm{r}}$ effect, are shown in Fig. 4 (b), and it is found that the neoclassical $\chi_{\mathrm{i}}$ is not increased with the $T_{\mathrm{i}}$ rise. Ripple transport is greatly enhanced without $E_{\mathrm{r}}$, and the positive- $E_{\mathrm{r}}$ effect suppresses the enhancement of ripple transport due to the $T_{\mathrm{i}}$ rise. Considering that the neoclassical $\chi_{\mathrm{i}}$ is not changed much by applying ECRH, the $T_{\mathrm{i}^{-}}$ rise is thought to be caused by the reduction of anomalous transport. 

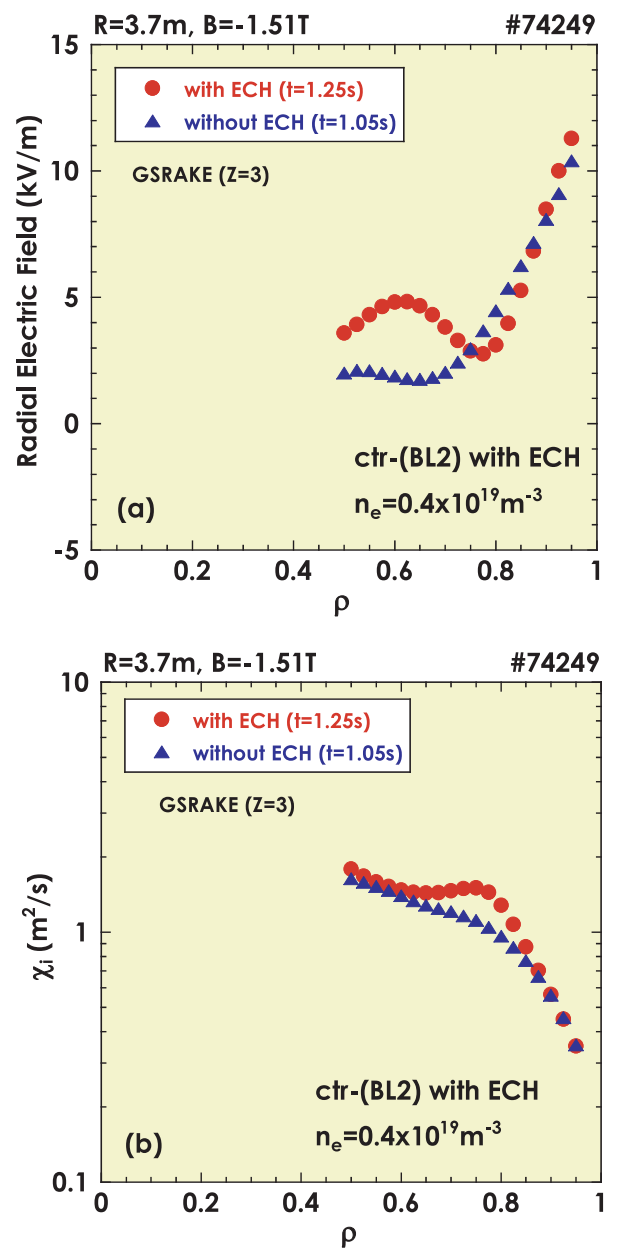

Fig. 4 Results of the neoclassical calculation using the GSRAKE code for the plasmas with and without the ECRH superposition shown in Fig. 2 (a). Radial electricfield profiles and (b) ion thermal-diffusivity profiles.

\section{Discussion}

In previous investigations on the electron-ITB in LHD, which have been reported in Refs. [3-6], no increase in $T_{\mathrm{i}}$ was observed in the CERC plasmas. In those experiments, only high-energy negative-NBI heating was used in the target plasmas for the ECRH superposition, and the ion heating power ratio was much smaller than the electron heating one. In the present experiments, on the other hand, low-energy positive-NBI heating is used together with the negative-NBI in the target plasmas, and the ion heating power ratio is enhanced compared with the previous experiments. As a result, the change in $T_{\mathrm{i}}$ would be more sensitive to the change in ion transport, and thus, a $T_{\mathrm{i}}$ rise has been observed in the present experiments. In the previous experiments, the $T_{\mathrm{i}}$ measurement was performed by CXS in a poloidal line of sight with a high-energy beam with a small cross section for the measurement. In that case, the peripheral $T_{\mathrm{i}}$ would have an influence on the core $T_{\mathrm{i}}$ measurement under such an experimental condition, as the ECRH superposition causes the impurity pump-out effect. In the present experiments, CXS in a toroidal line of sight is employed with a low-energy beam with a larger cross section for the measurement. Thus, the $T_{\mathrm{i}}$-profile measurement should be improved for observation of the $T_{\mathrm{i}}$-change. Another hypothesis considers the density profile. There is a tendency that the density-profile hollowness is suppressed with low-energy NBI heating [13]. Although the increase in the $T_{\mathrm{e}} / T_{\mathrm{i}}$ ratio in the CERC plasmas could destabilize the ion temperature gradient (ITG) mode, the destabilization of the ITG mode could be suppressed due to this effect with low-energy NBI heating.

The $T_{\mathrm{e}} / T_{\mathrm{i}}$ ratio is one of the important parameters for ion transport. In tokamaks, it has been reported that increasing the $T_{\mathrm{i}} / T_{\mathrm{e}}$ ratio decreases electron and ion transport [18]. This is related to the ITG mode, and it is thought that an increase in the $T_{\mathrm{e}} / T_{\mathrm{i}}$ ratio leads to lowering the critical ion temperature gradient for the destabilization of the ITG mode. Considering that the electron confinement is improved in the LHD-CERC plasmas, in which the $T_{\mathrm{e}} / T_{\mathrm{i}}$ ratio increases, the same discussion as that for tokamaks would not be necessarily applied. However, an excess increase in the $T_{\mathrm{e}} / T_{\mathrm{i}}$ ratio would lead to the destabilization of the ITG mode, which would suppress the $T_{\mathrm{i}}$ increase. On the other hand, the density gradient would also be related to the ITG mode, and low-energy NBI heating, in which the density hollowness is suppressed, may play a role in stabilization of the ITG mode. In LHD, the $T_{\mathrm{i}}$ profile measurement by CXS is improved with the combination of the injected low-energy neutral beams and the toroidal line of sight for the measurement. The correlation between the $T_{\mathrm{e}} / T_{\mathrm{i}}$ ratio and ion transport in the CERC plasma is an important subject to be solved, and its investigation has just started with the results presented in this article with the improved CXS measurement.

$E_{\mathrm{r}}$ plays an important role in the confinement improvement, and it would also influence the direct loss of energetic ions, especially for the perpendicularly injected particles. It is predicted that the loss of energetic ions with a specific pitch angle is reduced with positive $E_{\mathrm{r}}$. In LHD, the behavior of energetic ions in the CERC plasmas is investigated with neutral particle analyzers; however, the reduction of the direct ion loss with ECRH superposition has not been observed definitely. Thus, although this $E_{\mathrm{r}}$ effect for energetic ions should be investigated further, enhancement of the NBI heating power due to the reduction of the direct ion loss is not considered with ECRH superposition in the present analyses.

As shown in Fig. 2 (c), toroidal rotation driven by ECRH superposition seems to be correlated with the $T_{\mathrm{i}}$ rise. The neoclassical calculation code for the toroidal viscosity is being developed for the LHD high- $T_{\mathrm{i}}$ plasmas; however, the neoclassical prediction for toroidal rotation is not available currently. In near future, the correlation between toroidal rotation and ion transport would be clarified theoretically, including the effect of $E_{\mathrm{r}}$ on the toroidal viscosity. Then, the analysis of the experimental observation would be more understandable. 


\section{Summary}

An increase in ion temperature has been observed in the NBI-heated plasmas with the superposition of centrally focused ECRH in LHD. The target plasma is produced with a high-energy NBI, and a low-energy NBI is also used for ion heating and a $T_{\mathrm{i}}$-profile measurement by CXS. By applying ECRH to the NBI plasma, an increase in $T_{\mathrm{e}}$ is observed with a steep $T_{\mathrm{e}}$-gradient. This indicates the formation of the electron-ITB, in which the improvement of electron transport is recognized in CERC. Therefore, it is thought that the $T_{\mathrm{i}}$ increase is observed in CERC conditions. The location of the $T_{\mathrm{i}}$ rise seems to be different from that of the $T_{\mathrm{e}}$ rise. The transport analysis shows that the ion transport improvement is ascribed to the reduction of anomalous transport. Neoclassical ambipolar calculation shows that $E_{\mathrm{r}}$ is positive at the location of the $T_{\mathrm{i}}$-rise. This suggests that ion transport is improved in the CERC plasma. Considering that the neoclassical ion thermal diffusivity is not changed much, the experimental ion transport is dominated by anomalous transport. Toroidal rotation is measured by CXS in a toroidal line of sight, and a correlation is observed between toroidal rotation and the $T_{\mathrm{i}}$-rise by applying ECRH.

The improvement of ion transport in CERC is a possible scenario for increasing the ion temperature in helical systems, and this is experimentally demonstrated in LHD.

\section{Acknowledgments}

The authors are grateful to the technical staff in LHD for the excellent operation of LHD and the NBI and ECRH systems. This work has been supported by the NIFS under NIFS07ULBB501.

[1] M. Yokoyama et al., Fusion Sci. Technol. 50, 327 (2006).

[2] M. Yokoyama et al., Nucl. Fusion 47, 1213 (2007).

[3] Y. Takeiri et al., Phys. Plasmas 10, 1788 (2003).

[4] T. Shimozuma et al., Plasma Phys. Control. Fusion 45, 1183 (2003).

[5] K. Ida et al., Phy. Rev. Lett. 91, 085003 (2003).

[6] Y. Takeiri et al., Fusion Sci. Technol. 46, 106 (2004).

[7] O. Motojima et al., Nucl. Fusion 47, S668 (2007).

[8] O. Kaneko et al., Proc. 16th Int. Conf. on Fusion Energy 1996 (Montreal, 1996) Vol. 3 (Vienna, IAEA) p. 539.

[9] Y. Takeiri et al., Nucl. Fusion 46, S199 (2006).

[10] M. Osakabe et al., Proc. 17th International Toki Conference, Toki, Japan, 2007, P2-079.

[11] Y. Takeiri et al., Nucl. Fusion 45, 565 (2005).

[12] Y. Takeiri et al., Nucl. Fusion 47, 1078 (2007).

[13] K. Nagaoka et al., Plasma Fusion Res. 3, S1013 (2008).

[14] M. Yoshinuma et al., Plasma Fusion Res. 3, S1014 (2008).

[15] S. Kubo et al., Plasma Phys. Control. Fusion 47, A81 (2005).

[16] M. Osakabe et al., Rev. Sci. Instrum. 72, 586 (2001).

[17] C.D. Beidler and W. D. D'haeseleer, Plasma Phys. Control. Fusion 37, 463 (1995).

[18] C.C. Petty et al., Phy. Rev. Lett. 83, 3661 (1999). 